\title{
Ultrastructure of developmental stages of Hemolivia mariae (Apicomplexa: Haemogregarinidae), natural parasite of the Australian sleepy lizard, in experimentally infected deviant hosts
}

\author{
Ilan Paperna ${ }^{1}$ and Catherine J. Smallridge ${ }^{2}$ \\ ${ }^{1}$ Department of Animal Sciences, Faculty of Agriculture of the Hebrew University of Jerusalem, Rehovot, 76100, Israel; \\ ${ }^{2}$ School of Biological Sciences, Faculty of Science and Engineering, Flinders University of South Australia, GPO Box 2100, \\ Adelaide, South Australia 5001, Australia
}

Key words: Haemogregarinidae, Hemolivia mariae, ultrastructure, merogony, gametocytes, wall development, osmiophilic bodies

\begin{abstract}
Mabuya vitatta (Olivier) (Scincidae) and Agama stellio (L.) (Agamidae) were infected with Hemolivia mariae Smallridge et Paperna, 1997 by ingestion of tick viscera from Amblyomma limbatum Neumann, fed as nymphs on naturally infected Australian sleepy lizards, Tiliqua rugosa Gray. The unnatural infection apparently interfered with the developmental schedule of the parasites. Transmission electron microscopic images of merogonic stages were obtained, as well as images of early developing gametocytes. Tissue and intraerythrocytic meronts were bound by a hardened wall. Intraerythrocytic gametocytes were lodged in a parasitophorous vacuole, which was filled with granular material, and were bound by a twomembrane wall. Small and large osmiophilic bodies were located in a sub-pellicular position. With differentiation, the wall membranes tightened with the parasitophorous vacuole wall, and the osmiophilic bodies disappeared. The outer parasite membrane consolidated into a thick encasing with distinct sutures. Late infection in $A$. stellio comprised gametocytes only.
\end{abstract}

Hemolivia mariae Smallridge et Paperna, 1997 is a natural parasite of the Australian sleepy lizard Tiliqua rugosa Gray and is transmitted via the tick Amblyomma limbatum Neumann (Smallridge and Bull 1999). Electron microscopic studies were carried out on the blood stages (gametocytes) and the stages developing in ticks (Smallridge and Paperna 1997, 2000a, b). There are several available ultrastructural accounts on merozoites and dividing meronts of Hepatozoon spp. (Stehbens and Johnston 1968, Drolesky et al. 1993, Smith and Desser 1998, Smith et al. 2000). Most gametocytes described were mature or sub-mature (Baker and Lainson 1967, Stehbens and Johnston 1967, Bashtar et al. 1984, Nadler and Miller 1984, Paterson et al. 1988). The scanty appearance of exoerythrocytic stages of $H$. mariae in the natural lizard host impeded attempts to study their fine structure. Experimental infection of $H$. mariae in unnatural hosts of the natural host family (Scincidae) and also of another lizard family (Agamidae) produced aberrant infections that amplified and prolonged the developmental schedules of both merogonic and early gametocytic stages. In this communication, we present a fine structural study of developing merogonic and gamogonic stages as obtained from experimental infections in unnatural host species.

\section{MATERIALS AND METHODS}

Laboratory-reared Amblyomma limbatum, fed as nymphs on infected sleepy lizards (Tiliqua rugosa) were the source of infection 30-44 days later. Dissected tick viscera containing sporocysts were fed to Mabuya vitatta (Olivier) and Agama stellio (L.). The course of infection in these lizards is to be described elsewhere (Smallridge and Paperna, unpublished) One $M$. vitatta that became moribund 41 days post infection (p.i.) was euthanised. Clumps of coagulated blood, liver and lungs were taken for transmission electron microscopy (TEM). The A. stellio was killed at 5 months p.i. and its blood and liver were taken for TEM. The tissues from both lizards were fixed in $2.5 \%$ glutaraldehyde in $0.1 \mathrm{M}$ sodium cacodylate buffer ( $\mathrm{pH} 7.4$ ) for $24 \mathrm{~h}$ at $4^{\circ} \mathrm{C}$. They were then rinsed three times in cacodylate buffer, post-fixed for $1 \mathrm{~h}$ in $1.0 \%$ osmium tetroxide in the same buffer, rinsed again with buffer, dehydrated in a graded alcohol series and embedded in Agar 100 resin (Agar Co., UK). Thin sections, cut on a Reichert-Jung Ultracut E microtome with a diamond knife, were collected onto coated and uncoated grids, stained with $8 \%$ aqueous uranyl acetate for $30 \mathrm{~min}$ in darkness, and then stained for $7 \mathrm{~min}$ with Reynold's lead citrate. The thin sections were examined in a JEOL $100 \mathrm{CX}$ transmission electron microscope.

\section{RESULTS}

\section{Stages in the tissues of Mabuya vitatta}

The anterior end of a single merozoite, within a parasitophorous vacuole (PV) inside a degrading cell (possibly a macrophage), contained a visible conoid, rhoptries and numerous micronemes. Of the variety of subcellular structures present, the most conspicuous were amylopectin granules and mitochondria (Fig. 1). 
Several phases of meront division were observed lodged in PV in endothelial cells (Figs. 2-4). Undivided meronts, as well as multinucleate and divided meronts prior to merozoite separation, were bound by a rigid wall as evident from signs of shrinkage (Figs. 2, 3), and a suture was detected on this encasement (Fig. 4). During the maturation process, amylopectin bodies became confined to the meront residuum (Fig. 4). The outlines of the merozoites became conspicuous and contained a large nucleus associated with a Golgi complex (Fig. 4). The separating merozoites contained large mitochondria, well-demarcated rough endoplasmic reticulum (ER), and amylopectin bodies (Fig. 5). In addition to micronemes, the cytoplasm contained a few osmiophilic bodies (which are numerous in later stages), recognised by their drainage ducts and many electron-dense globules which were distinct from the micronemes (Fig. 5). Separating merozoites still within their parent $\mathrm{PV}$, showed a similar range of organelles, most conspicuously large mitochondria (Fig. 6). The meront residuum still remained loaded with ER elements, amylopectin granules and a few lipid inclusions. The meront wall persisted to the final stage of the division.

\section{Stages in the erythrocytes of Mabuya vitatta}

Images of intraerythrocytic merogonic stages included a meront prior to division (Fig. 7) and a divided meront (Fig. 8). Similar to the tissue stages, both were encased in a hardened wall as evidenced by shrinkage, and demonstrated a similar fine structural character. The former had a large nucleus, occupying about one third of the cell, along with numerous amylopectin granules, scattered ribosomes and poorly preserved mitochondria (Fig. 7). The merozoites in the divided meront each had a large nucleus situated in closer proximity to the apical end which was marked by the presence of micronemes (Fig. 8).

The exoerythrocytic merozoites appear to become the future gametocytes (Fig. 9). Bound by a pellicle, they contained rhoptries at the anterior end, a variable number of sub-pellicular osmiophilic bodies, and also an adnuclear or paragolgi organelle. Vesicles of a granular substance appeared to be connected to the pellicle via ducts. Amylopectin granules were few, but lipid inclusions were prominent. At the anterior apical end, it was possible to detect an elaborate microtubular complex (Fig. 10), a conoid within a polar ring and a rhoptry extending into the conoid zone (Fig. 11).

Intraerythrocytic gametocytes lodged within a PV wall (w1) were bound by a double membrane wall (w23 ), with the wide lumen filled with granular material between the outermost (w1) and the middle layer (w2) representing the PV (Figs. 12, 13). The apical end showed sub-pellicular microtubules embedded in an electron-dense substance, with an adjacent array of rhoptries and lesser electron-dense bodies, evidently micronemes (Fig. 14). The same concentration of rhoptries and micronemes was demonstrated in the anterior end of the gametocyte in Fig. 15. In the gametocyte, small and large osmiophilic bodies were located in a sub-pellicular position, sometimes revealing their drainage connection to the pellicle. Some of the larger electron-dense bodies could be, however, either rhoptry sections or "dense granules" (Fig. 13). The large nucleus contained patches of chromatin; other elements of the cytoplasm were scattered rough ER, some amylopectin bodies, lipid vacuoles, and mitochondria (Figs. 12, 13, 15).

With the advent of differentiation, the wall membranes tightened (Figs. 15, 16) and sometimes an additional membrane was added (Fig. 17). Such gametocytes had large mitochondria, and developed strongly demarcated ER, some of which, in close proximity to a large Golgi apparatus, seemed to be associated with forming amylopectin bodies (Fig. 16).

Encasement was preceded by the appearance of two thickenings on the middle membrane (w2), which became bi-laminated at each pole of the gametocyte. These marked the future sites of the sutures in the wall capsule of the mature gametocyte. At this stage, the gametocyte cytoplasm became densely filled with rough ER while only a few osmiophilic bodies remained. They also contained many lipid vacuoles but only a few amylopectin granules (Fig. 18). The membrane, w2, thickened and became consolidated into a thick encasing wall overlaid by a fine glycocalyx or a fine granular layer (Fig. 19). With the final solidification of the encasement, there was evidence of reduced permeability that resulted in an increased impact of processing distortion. The encasement was interrupted by distinct sutures. The nucleus was still visible at one end of the gametocyte as well as, in the cytoplasm, rough ER, and a few amylopectin granules (Fig. 20).

\section{Stages in the erythrocytes of Agama stellio}

In $A$. stellio, the infection predominantly comprised gametocytes. These occurred both in the circulatory blood in erythrocytes (Fig. 21), and accumulated in aggregated melanomacrophages in the liver (Fig. 22). In both locations the gametocytes were encased in a hard wall with a suture at each end. Very few encapsulated divided meronts were traced in melanomacrophages alongside gametocytes.

\section{DISCUSSION}

The unnatural infection apparently interferes with the developmental schedule of the parasites. As a result, we were able to obtain images of merogonic stages as well as of early developing intraerythrocytic gametocytes.

In TEM images of eimeriid coccidians, the parasitophorous wall is usually widely separated from the parasite boundary (Entzeroth et al. 1998). The tightened arrangement of the wall layers of the intraerythrocytic 


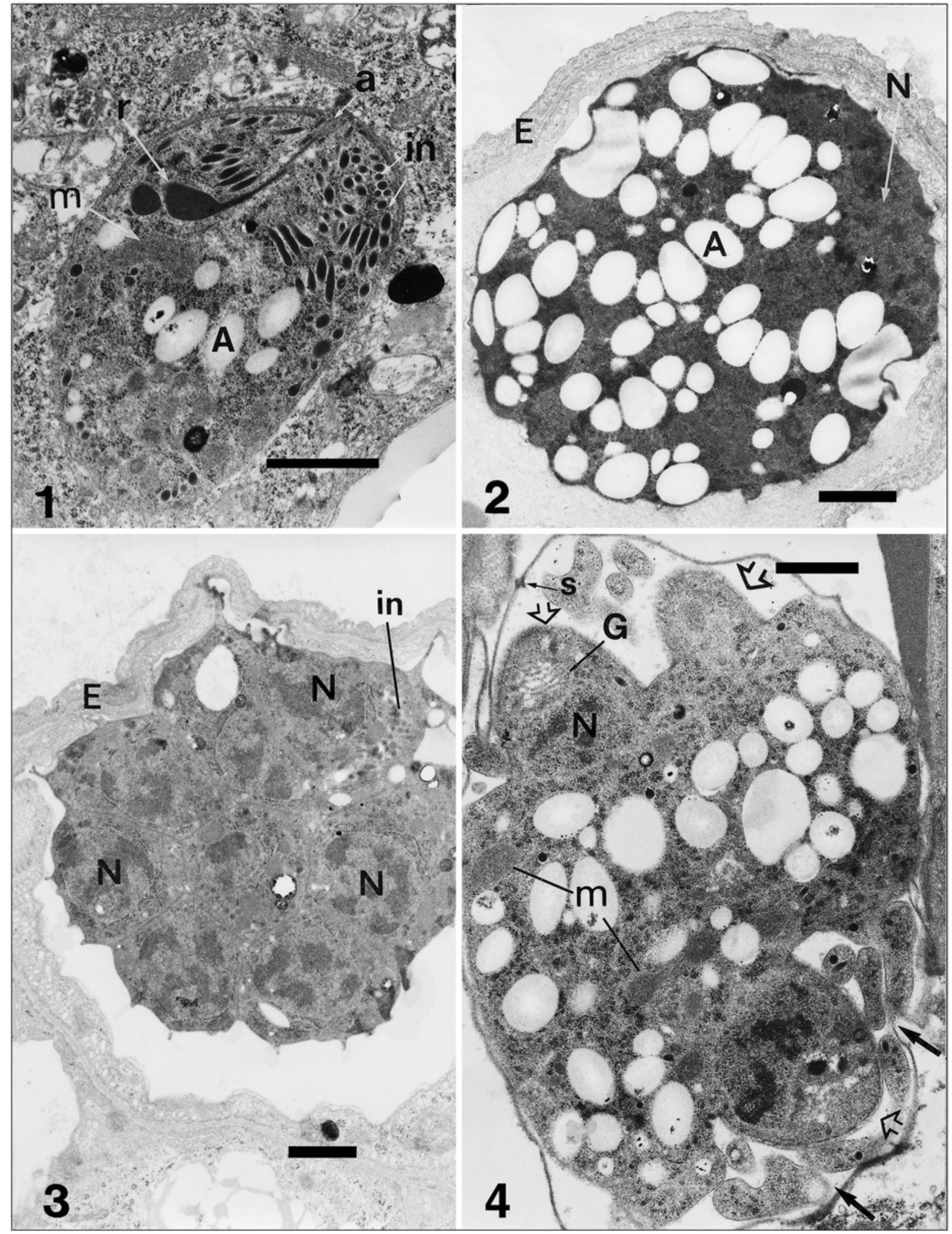

Figs. 1-4. Merogonic stages of Hemolivia mariae in tissues of Mabuya vitatta. Fig. 1. Early merozoite in liver (m mitochondrion, $\mathrm{A}$ - amylopectin bodies, $\mathrm{r}$ - rhoptries, a - apical complex, in - micronemes). Fig. 2. Early dividing meront inside an endothelial cell (E), ( $\mathrm{N}$ - nucleus, A - amylopectin bodies). Fig. 3. Encased, divided meront inside an endothelial cell (E), (N - merozoite nuclei, in - micronemes). Fig. 4. Merozoite primordia (open arrows), containing a nucleus (N) and a Golgi complex (G) emerging from the meront body filled with amylopectin bodies. The meront wall shows a suture (s). Small cytoplasmic enclaves are marked with solid arrows, some contain micronemes $(\mathrm{m}-$ mitochondria). Scale bars $=1 \mu \mathrm{m}$. 


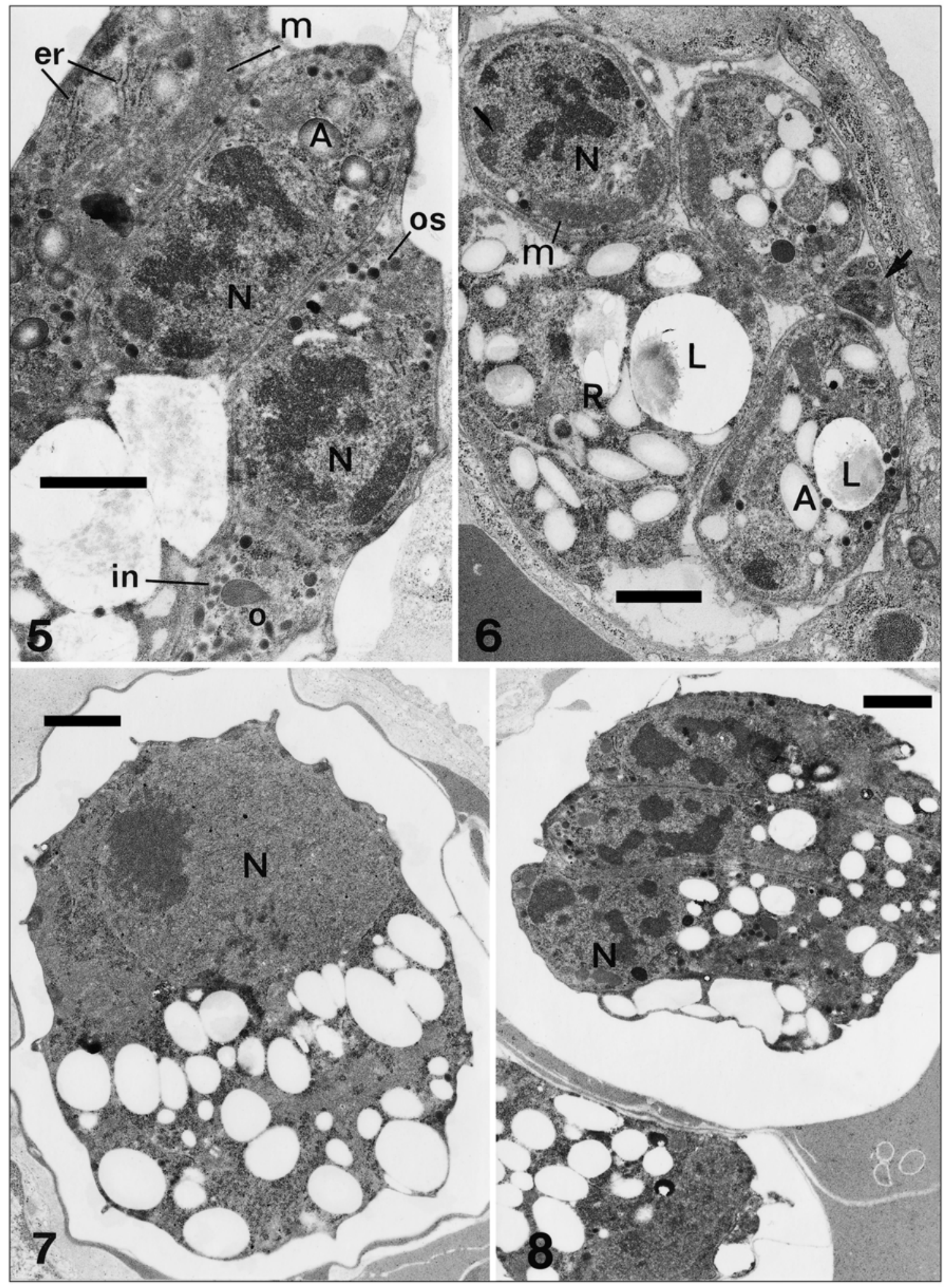

Figs. 5-8. Merogonic stages of Hemolivia mariae in tissues of Mabuya vitatta. Fig. 5. Emerging merozoites showing endoplasmic reticulum (er) and amylopectin bodies (A). Osmiophilic bodies (o) drained by ducts and smaller electron-dense globules (os). Fig. 6. Merozoites and meront residuum (R) still inside a parasitophorous vacuole in an endothelial cell (N nucleus, $\mathrm{m}$ - mitochondria, L - lipid vacuole, A - amylopectin body, arrow - cytoplasmic enclaves). Fig. 7. Intraerythrocytic, encased meront before division ( $\mathrm{N}$ - nucleus). Fig. 8. Intraerythrocytic merozoite formation ( $\mathrm{N}$ - nucleus). Scale bars $=1 \mu \mathrm{m}$. 


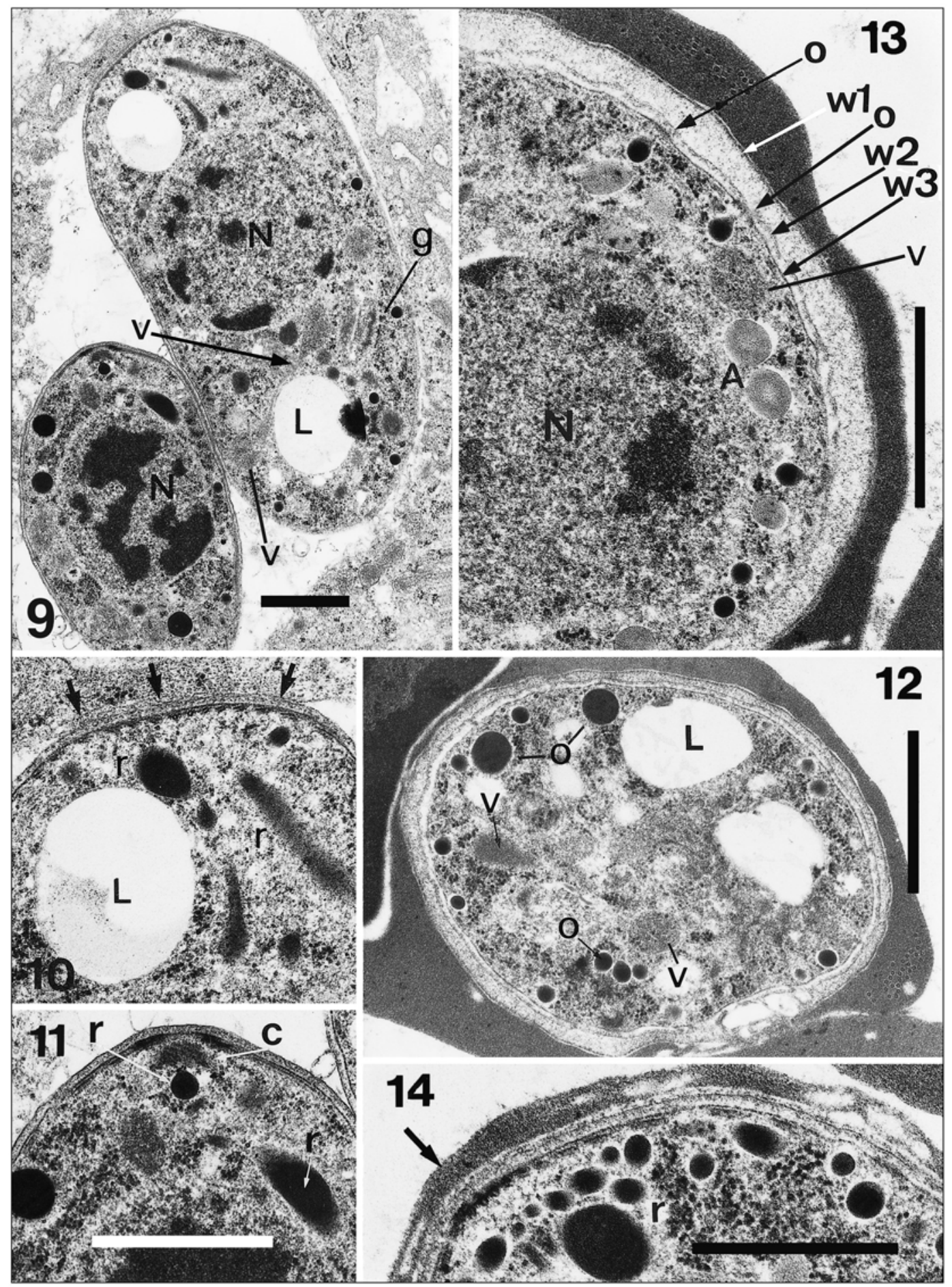

Figs. 9-14. Gamogonic stages of Hemolivia mariae in tissues of Mabuya vitatta. Fig. 9. Merozoites (presumed pre-gametocytes) showing apical zones with rhoptries and other cytoplasmic organelles $(\mathrm{N}$ - nucleus, $\mathrm{L}$ - lipid vacuole, g-adnuclear organelle, $\mathrm{v}$ - interconnected vesicles). Fig. 10. Enlargement of an apical zone showing microtubular elements (arrows), ( $\mathrm{r}-\mathrm{rhoptries,} \mathrm{L}-$ lipid vacuole) (same scale as in Fig. 11). Fig. 11. Enlarged view of an apical zone with conoid (c), an adjunct and other rhoptries (r). Fig. 12. Young intraerythrocytic gametocytes (o - small and large osmiophilic bodies, v-vesicles, L - lipid vacuole). Fig. 13. Enlarged view of a young intraerythrocytic gametocyte showing parasitophorous vacuole (PV) and parasite boundary elements: w1 - PV wall, w2 - outer parasite wall, w3 - inner parasite wall (o - osmiophilic bodies, v - vesicle, A - amylopectin bodies, $\mathrm{N}$ - nucleus). Fig. 14. Details of gametocyte apical zone: microtubule-pellicle junction (arrow), (r - rhoptry). Scale bars $=1 \mu \mathrm{m}$. 


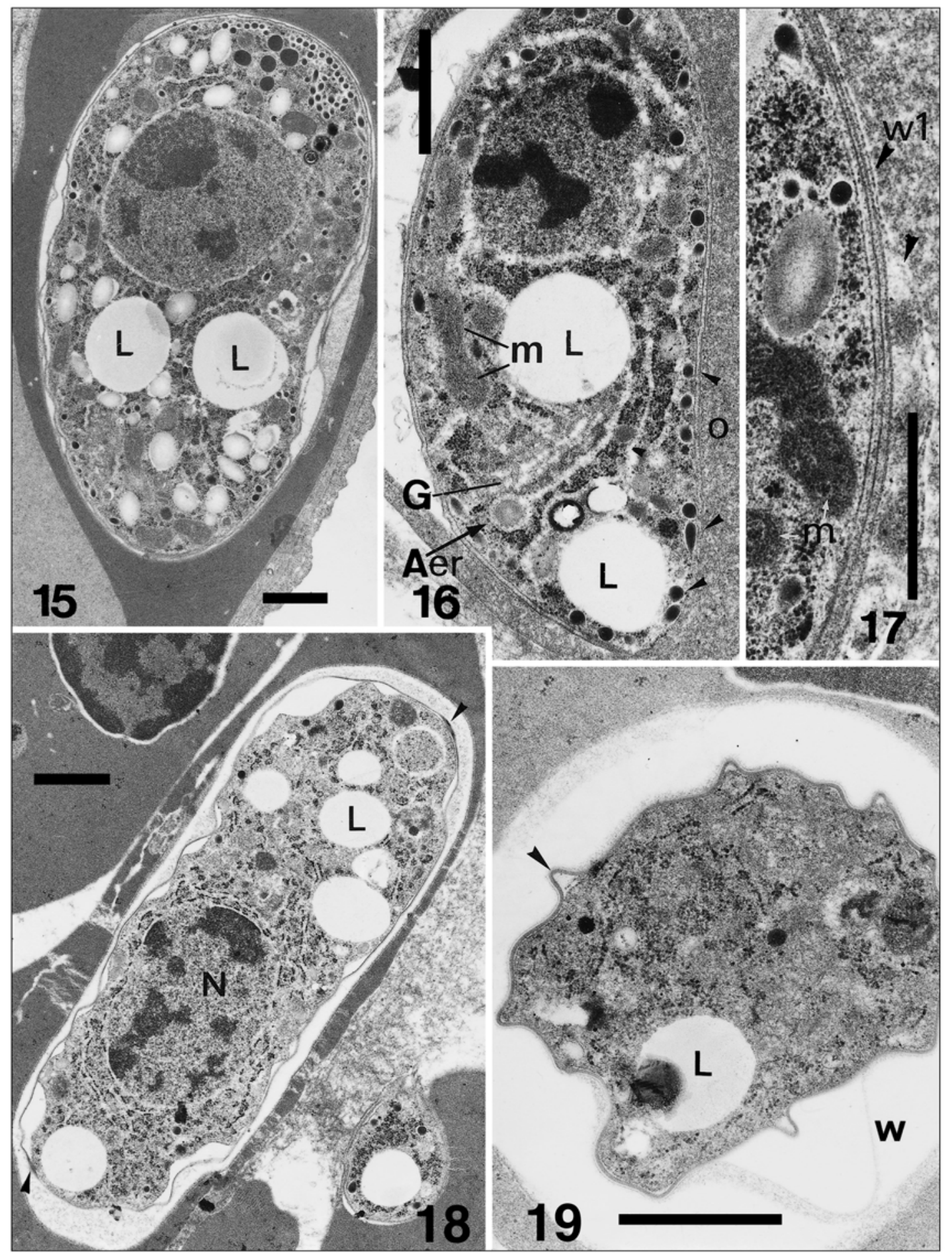

Figs. 15-19. Intraerythrocytic stages of Hemolivia mariae in tissues of Mabuya vitatta. Fig. 15. Gametocytes showing progressive depletion of the granular content of the parasitopohorous vacuole (PV) (L - lipid vacuole). Fig. 16. Premature gametocyte showing Golgi apparatus $(\mathrm{G})$ and conspicuous endoplasmic reticulum (Aer) with a site of apparent amylopectin body formation ( $\mathrm{m}$ - mitochondria, L - lipid vacuole, o and arrowheads - osmiophilic bodies). Fig. 17. Tightened membranous boundary of a premature gametocyte ( $\mathrm{m}-$ mitochondria, w1 - PV boundary). Fig. 18. Gametocyte at onset of encasement; cytoplasm is loaded with rough ER ( $\mathrm{L}$ - lipid vacuole, $\mathrm{N}$ - nucleus), arrowheads mark the forming sutures. Fig. 19. Gametocyte with gradually hardening wall (arrowhead), ( w - surface coat of either glycocalyx or fine granular matrix, L - lipid vacuole). Scale bars $=1 \mu \mathrm{m}$. 


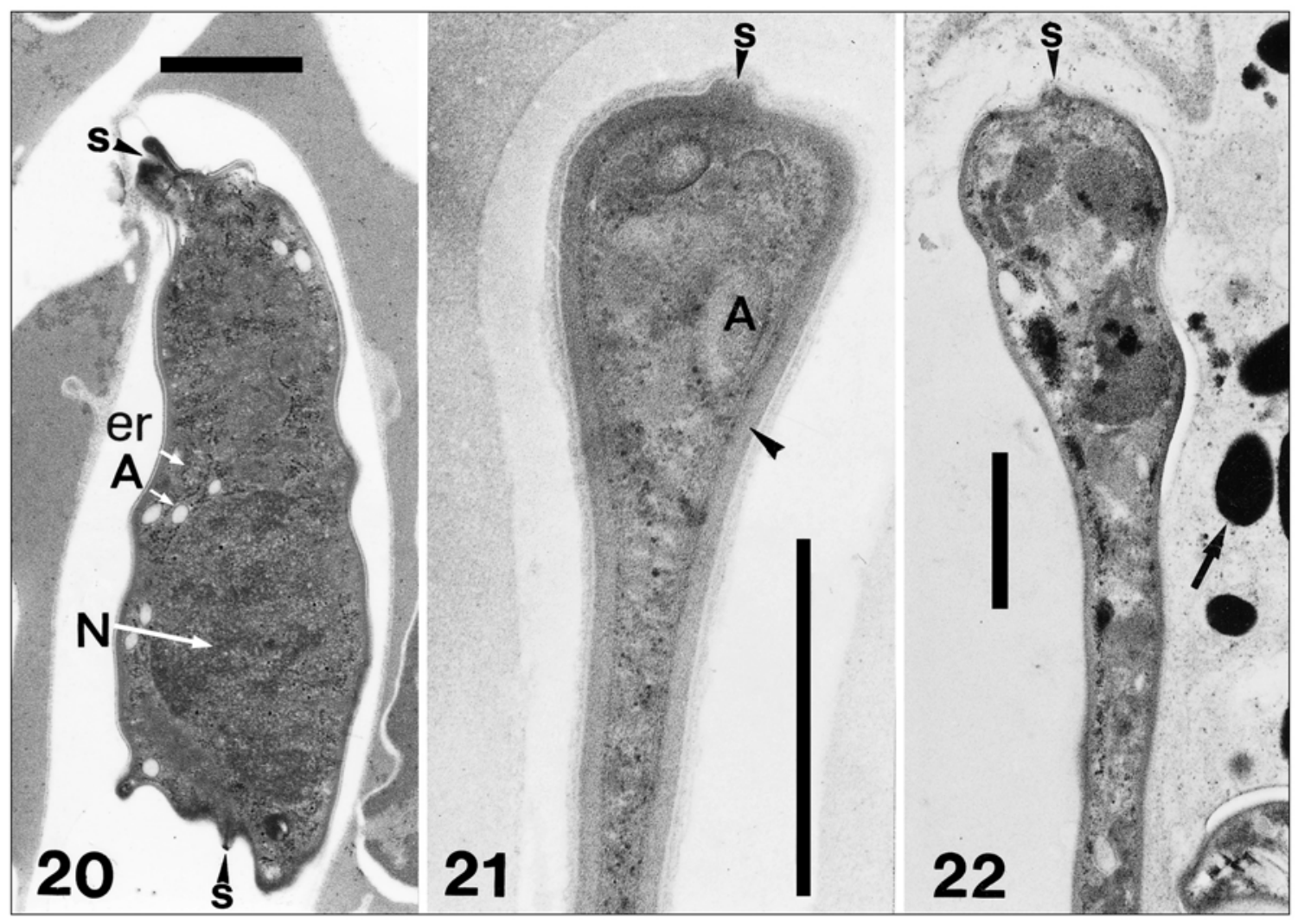

Figs. 20-21. Gametocytes of Hemolivia mariae in Mabuya vitatta and Agama stellio. Fig. 20. Gametocyte in M. vitatta erythrocyte, encased in hardened wall with distinct sutures (s), ( N - nucleus, A - amylopectin bodies, er - endoplasmic reticulum). Fig. 21. Intraerythrocytic gametocyte of $H$. mariae in A. stellio (A - amylopectin body, s - suture, arrowhead hardened wall). Fig. 22. Gametocyte in melanomacrophage centre in liver of A. stellio (s - suture, arrow - melanin granule). Scale bars $=1 \mu \mathrm{m}$.

gametocytes with the closely applied parasitophorous vacuole wall, is reminiscent of the cell boundary seen in intraerythrocytic stages of haemosporidians. This, together with the presence of the osmiophilic bodies (Aikawa et al. 1969), might hint at some phylogenetic relationship between haemogregarines and haemosporidians. This complex wall system has not been yet reported in other haemogregarines, although some published images of intraerythrocytic stages of Hepatozoon (Nadler and Miller 1984, Paterson et al. 1988) do suggest the similar development of a complex wall system.

The granular contents of the parasitophorous vacuole depletes with further differentiation of the gamont and results in a tight multilayered membranous investment. The parasite's outer layer gives rise to the hard wall.

Other fine structural details observed in this study have already been described in the literature on hemogregarine ultrastructure (Bashtar et al. 1984, Paterson et al. 1988, Smith and Desser 1998). Some confusion may arise from the interpretation of the observed electron-dense organelles. We have no doubt about the presence of osmiophilic bodies which, similar to the ones seen in haemosporidians, show the drainage ducts to the pellicle. However, those in which this duct is concealed could be mistaken for either rhoptries or "dense granules," which are present in a wide range of coccidial organisms and are involved in the cell invasion process (Entzeroth et al 1998). We are not certain about the function of the osmiophilic bodies. They are present in mature gametocytes of haemosporidians (Aikawa et al. 1969, Paperna and Boulard 2000); in Hemolivia mariae gametocytes they disappear with the onset of maturity. Osmiophilic bodies were seen also in the recently studied Hemolivia stellata (Boulard et al. 2001). Siddall and Desser (1990, 1992) reported micronemes and dense organelles with peduncles extending to the plasmalemma in sporozoites of Haemogregarina balli and H. myxocephali. They regarded them homologues of dense bodies of coccidians involved, through exocytosis in the invasion of host cells (Entzeroth et al. 1998). The vesicles, which are apparently interconnected by ducts, are too vaguely outlined for any interpretation of their identity. Association between apparently forming amylopectin bodies and ER ducts has been observed also in tissue stages of H. stellata (Boulard et al. 2001) 
Encasement in the gametocytes is associated with the long-lived nature of this stage in the circulatory blood, which increases the probability of successful vectormediated transmission. By the same strategy, meront encasement may also facilitate longer persistence as source for gametocyte relapses. The extent of gametocyte encasement varies among the different haemogregarine taxa, reaching its extreme in species of Hemolivia (Smallridge and Paperna 2000a, Boulard et al. 2001).
Sporozoites of the genera Lankesterella and Schellackia accumulate and survive in the melanomacrophage centres prior to their release into the circulatory blood (Paperna and Ostrovska, 1989, Paperna and Martin, 2001). However, H. mariae gametocytes accumulating in the melanomacrophage centres in A. stellio seem to be on way to destruction rather than being stored for subsequent transmission via predation.

\section{REFERENCES}

AIKAWA M., HUFF C.G., SPRINZ H. 1969: Comparative fine structure of the gametocytes of avian, reptilian and mammalian malarian parasites. J. Ultrastruct. Res. 26: 316-331.

BAKER J.R., LAINSON R. 1967: The fine structure of the gametocytes of an adeleine haemogregarine. J. Protozool. 14: 233-238.

BASHTAR A.-R., GHAFFAR F.A., MEHLHORN H. 1984: Hepatozoon aegypti nov. sp. 2. Electron microscope studies on the erythrocytic stages and schizogony inside the snake, Spalerosophis diadema. Z. Parasitenkd. 70: 4352.

BOULARD Y., PAPERNA I., PETIT G., LANDAU I. 2001: Ultrastructure of developmental stages of the haemogregarine Hemolivia stellata in the cane toad Bufo marinus and its vector tick Amblyomma rotondatum. Parasitol. Res. 87: 598-604.

DROLESKY R.E., MERCER S.H., DeLOACH J.R., CRAIG T.M. 1993: Ultrastructure of Hepatozoon canis in the dog. Vet. Parasitol. 50: 83-99.

ENTZEROTH R., MATTIG F.R., WERNER-MEIER R. 1998: Structure and function of the parasitophorous vacuole in Eimeria species. Int. J. Parasitol. 28: 10151018.

NADLER S.A., MILLER J.H. 1984: Fine structure of Hepatozoon mocassini (Apicomplexa, Eucoccidiorida) gamonts and modifications of the infected erythrocyte plasmalemma. J. Protozool. 32: 275-279.

PAPERNA I., BOULARD Y. 2000: Fine structure of gametocytes in five species of Haemoproteus (Haemosporidia) from geckos and agamid lizards. Zoosystema 22: 443-457.

PAPERNA I., MARTIN C. 2001: The development and fine structure of Lankesterella cf. dicroglossi (Apicomplexa: Lankesterellidae) infecting frogs in Niger, West Africa. Folia Parasitol. 48: 178-186.

PAPERNA I., OSTROVSKA K. 1989: Ultrastructural studies on sporozoite stages of Schellackia cf. agamae from liver and blood of the starred lizard Agama stellio. Int. J. Parasitol. 19: 13-19.

PATERSON W.B., DESSER S.S., BARTA J.R. 1988: Ultrastructural features of the apical complex, pellicle and membranes investing gamonts of Haemogregarina magna (Apicomplexa: Adeleina). J. Protozool. 35: 73-80.
SIDDALL M.E., DESSER S.S. 1990: Gametogenesis and sporogonic development of Haemogregarina balli (Apicomplexa: Adeleina: Haemogregarinidae) in the leech Placobdella ornata. J. Protozool. 37: 511-520.

SIDDALL M.E., DESSER S.S. 1992: Ultrastructure of gametogenesis and sporogony of Haemogregarina (sensu lato) myxocephali (Apicomplexa: Adeleina) in the marine leech Malmiana scorpii. J. Parasitol. 39: 545-554.

SMALLRIDGE C.J., BULL C.M. 1999: Transmission of the blood parasite Hemolivia mariae between its lizard and tick hosts. Parasitol. Res. 85: 858-863.

SMALLRIDGE C.J., PAPERNA I. 1997: The tick-transmitted haemogregarinid of the Australian sleepy lizard Tiliqua rugosa belongs to the genus Hemolivia. Parasite 4: 359363.

SMALLRIDGE C.J., PAPERNA I. 2000a: Ultrastructure of Hemolivia mariae gamonts in the blood of the lizard Tiliqua rugosa and their development to oocyst stage in the tick Amblyomma limbatum. Parasitol. Res. 86: 563569.

SMALLRIDGE C.J., PAPERNA I. 2000b: Ultrastructure studies on post-oocyst development of the lizard hemogregarine Hemolivia mariae in the tick Amblyomma limbatum. Parasitol. Res. 86: 467-471.

SMITH T.G., DESSER S.S. 1998: Ultrastructural features of cystic and merogonic stages of Hepatozoon sipedon (Apicomplexa: Adeleiorina) in Northern leopard frogs (Rana pipiens) and Northern water snakes (Nerodia sipedon) from Ontario, Canada. J. Euk. Microbiol. 45: 419-425.

SMITH T.G., KIM B., HONG H., DESSER S.S. 2000: Intraerythrocytic development of species of Hepatozoon infecting ranid frogs: evidence for convergence of life cycle characteristics among apicomplexans. J. Parasitol. 86: 451-458.

STEHBENS W.E., JOHNSTON M.R.L. 1967: The ultrastructure of a haemogregarine parasitic in Gehyra variegata (Dumeril \& Bibron, 1836). Parasitology 57: 251-261.

STEHBENS W.E., JOHNSTON M.R.L. 1968: Cystic bodies and schizonts associated with a haemogregarine (Sporozoa) parasitic in Gehyra variegata (Reptilia: Gekkonidae). J. Parasitol. 54: 1151-1165. 